\title{
Behavioural interventions targeting physical activity improve psychocognitive outcomes in COPD
}

\author{
Kim L. Lavoie ${ }^{1,2}$, Maria Sedeno $\mathbb{1}^{3}$, Alan Hamilton ${ }^{4}$, Pei-Zhi Li ${ }^{3}$, \\ Dorothy De Sousa ${ }^{4}$, Thierry Troosters ${ }^{5}$, François Maltais ${ }^{6}$ and Jean Bourbeau $\mathbb{1}^{3}$
}

Affiliations: ${ }^{1}$ Montreal Behavioural Medicine Centre, Research Centre, CIUSSS-NIM Hôpital du Sacré-Coeur de Montreal, Montreal, Canada. ${ }^{2}$ Dept of Psychology, University of Québec at Montreal (UQAM), Montreal, Canada. ${ }^{3}$ Respiratory Epidemiology Clinical Research Unit, Research Institute of the McGill University Health Centre, Montreal, Canada. ${ }^{4}$ Boehringer Ingelheim (Canada) Ltd, Burlington, Canada. ${ }^{5} \mathrm{KU}$ Leuven, Dept of Rehabilitation Sciences, Pulmonary Rehabilitation and Respiratory Division, University Hospital Leuven, Leuven, Belgium. ${ }^{6}$ Centre de Recherche, Institut universitaire de cardiologie et de pneumologie de Québec, Université Laval, Québec, Canada.

Correspondence: Kim L. Lavoie, Dept of Psychology, UQAM, CP 8888, Succursale Centre-Ville, Montreal, Québec, Canada, H3C 3P8. E-mail: lavoie.kimauqam.ca

ABSTRACT This study explored the impact of a self-management behaviour modification (SMBM) programme with/without bronchodilators and with/without exercise training (ExT) to improve daily physical activity on psychological and cognitive outcomes in COPD patients as a secondary analysis of the PHYSACTO trial.

A 12-week, four-group, randomised, partially double-blind, placebo-controlled, parallel-group trial of SMBM in addition to tiotropium $5 \mu \mathrm{g}$, tiotropium/olodaterol $5 / 5 \mu \mathrm{g}$, tiotropium/olodaterol $5 / 5 \mu \mathrm{g}$ plus ExT, or placebo was conducted in 304 patients. Outcomes included anxiety (Hospital Anxiety and Depression Scale (HADS)-A), depression (HADS-D and Patient-Health Questionnaire (PHQ)-9) and cognitive function (Montreal Cognitive Assessment (MoCA)).

All outcomes showed statistically and clinically significant improvements after 12 weeks independent of treatment group. However, greater improvements in HADS-A and MoCA were seen in patients who exhibited greater increases in physical activity and exercise capacity, respectively, whereas greater improvements in HADS-D and PHQ-9 were seen in patients who exhibited increases in either physical activity or exercise capacity.

The results indicate that SMBM with/without bronchodilators or ExT was associated with improved psychological and cognitive functioning. Anxiety reduced with increased physical activity, cognitive function improved with increased exercise capacity, and depression reduced with increases in either physical activity or exercise capacity. Interventions that increase daily physical activity or exercise capacity may improve psychological and cognitive outcomes in COPD.

@ERSpublications

Behavioural modification adjunct to bronchodilator therapy and exercise training to increase exercise capacity and physical activity can also be beneficial for improving anxiety, cognitive function and depression in patients with COPD http://bit.ly/33ZufNM

Cite this article as: Lavoie KL, Sedeno M, Hamilton A, et al. Behavioural interventions targeting physical activity improve psychocognitive outcomes in COPD. ERJ Open Res 2019; 5: 00013-2019 [https://doi.org/10.1183/23120541.00013-2019].

This study is registered at www.clinicaltrials.gov with identifier number NCT02085161. The sponsor of the PHYSACTO trial (Boehringer Ingelheim) is committed to responsible sharing of clinical study reports, related clinical documents and patient-level clinical study data. Researchers are invited to submit inquiries via the Clinical Study Data Request website (https://www.clinicalstudydatarequest.com).

Received: 18 April 2019 | Accepted after revision: 16 Aug 2019

Copyright $\odot$ ERS 2019. This article is open access and distributed under the terms of the Creative Commons Attribution Non-Commercial Licence 4.0. 


\section{Introduction}

Patients with COPD experience a progressive worsening of activity-related breathlessness, which can severely impact their daily lives [1-3]. Due to the discomfort experienced, patients often avoid engaging in physical activity. This has been associated with further deconditioning, decreased exercise capacity and more rapid disease progression, often resulting in an impaired ability to engage in daily activities and significant psychological distress [4].

The clinical management of COPD includes a combination of pharmacotherapy and pulmonary rehabilitation [5]. However, the effectiveness of these treatments relies upon the patient's ability to understand and adhere to prescribed interventions, which may be compromised in patients with COPD due to psychological factors and cognitive limitations. Poor psychosocial adaptation to having COPD, feelings of helplessness and fear/panic in response to breathlessness, and exertional breathlessness that is not relieved by medication are thought to contribute to heightened levels of psychological distress in this population [6, 7]. Symptoms of both depression and anxiety have been associated with diminished exercise performance, less participation in pulmonary rehabilitation and an increased risk of exacerbations [8-10].

Cognitive impairments have been shown to affect patients with COPD with a significantly higher frequency than is observed among age-matched controls $[11,12]$. Cognitive impairments in patients with COPD may result from both pulmonary (e.g. hypoxia, hypercapnia and exacerbations) and nonpulmonary factors (e.g. decreased physical activity) that tend to worsen as the disease progresses [13, 14]. The cognitive impairments observed tend to affect attention, working memory, executive function (judgement and reasoning), psychomotor speed, language skills and complex visual-motor processes [15, 16]. The presence of cognitive impairments has also been shown to interfere with several aspects of treatment adherence and self-management among patients with COPD, including inhaler compliance and participation in pulmonary rehabilitation $[17,18]$.

There is a need to explore treatments that could lead to improvements in psychological and cognitive functioning among patients with COPD. There is evidence linking exercise (any planned, structured and repetitive movement designed to improve or maintain fitness) and physical activity (any bodily movement leading to energy expenditure) to improvements in depression and anxiety in patients with psychiatric [19] and chronic disease [20]. Exercise and physical activity have also been associated with slower rates of cognitive decline and improvements in cognitive function in both healthy subjects [21] and those with chronic disease [22], as well as in those with mild cognitive impairment [23] and dementia [24]. A recent trial highlighted the positive effects of exercise training (ExT) on cognitive function in patients with COPD [25]. However, the relative impact of ExT versus engaging in regular daily activities on psychological and cognitive functioning in patients with COPD has not been explored.

The PHYSACTO trial assessed the effects of a multimodal approach to COPD treatment [26-28], focusing on whether tiotropium/olodaterol (T/O), with or without ExT, would improve exercise endurance time (EET) and physical activity compared with placebo in patients participating in an self-management behaviour modification (SMBM) programme (figure 1). Psychological variables (i.e. levels of depressive and anxiety symptoms, and rates of clinically significant depression), and cognitive function pre- and post-treatment were also measured. This substudy aimed to evaluate treatment effects on both psychological and cognitive functioning. We hypothesised that psychological and cognitive functioning would improve significantly from pre- to post-treatment in all treatment arms, with the greatest improvements seen in those also receiving ExT $(\mathrm{SMBM}+\mathrm{T} / \mathrm{O}+\mathrm{ExT})$. We expected improvements in

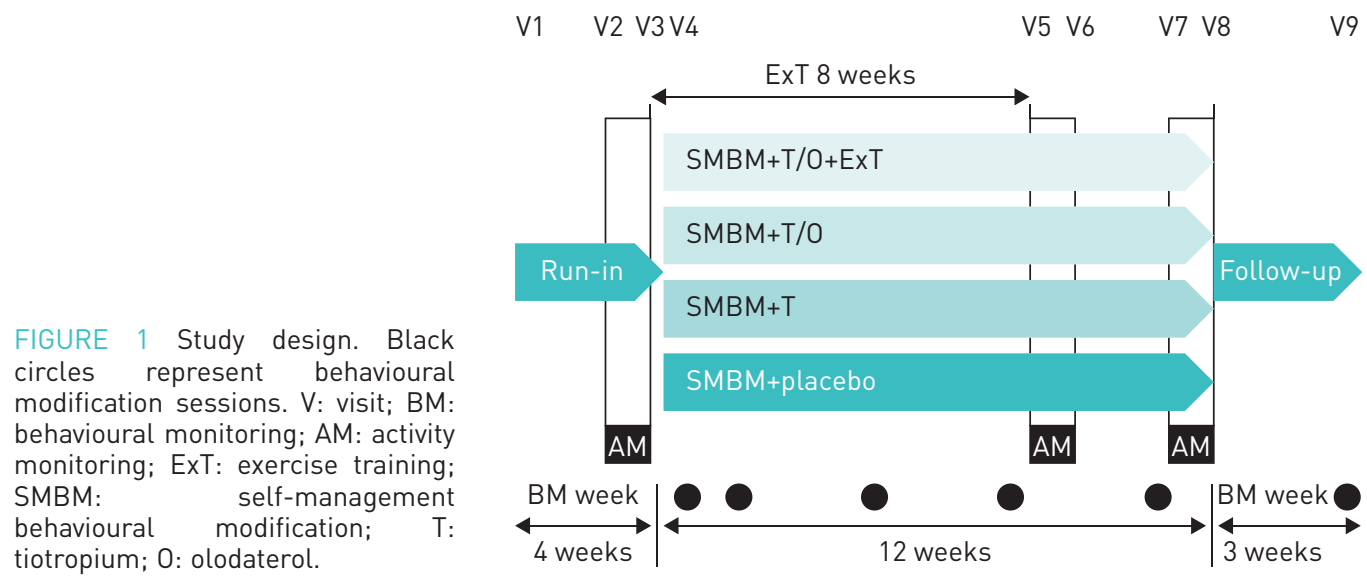


psychological and cognitive functioning would be positively correlated with actual/observed improvements in exercise capacity and physical activity.

\section{Methods}

Study design

Full details of the study design have been previously published [26-28]. PHYSACTO (study 1237.16; www. clinicaltrials.gov identifier number NCT02085161) was a 12-week, randomised, partially double-blind, placebo-controlled, parallel-group trial in patients with COPD. All pharmacological treatments were administered in a blinded manner, except for participants and staff in the group receiving ExT. The study was conducted in accordance with the principles of the Declaration of Helsinki and the International Conference on Harmonized Tripartite Guideline for Good Clinical Practice. The study complied with all Institutional Review Board, independent ethics committee, and national and international competent authority regulations. All patients were required to provide written, informed consent prior to participation.

\section{Interventions}

Details of the interventions have been published elsewhere [27]. Briefly, all patients participated in a 12-week SMBM programme adapted from the Living Well with COPD programme [29] delivered using a motivational communication style that aimed to increase patients' motivation and confidence in their ability to engage in higher levels of daily physical activity [26]. This programme was delivered by carefully selected case managers that received standardised training from and supervision by the global behaviour modification team (K.L. Lavoie, J. Bourbeau and M. Sedeno). Case managers used evidence-based behaviour change techniques (e.g. goal setting, problem solving and positive reinforcement), which were delivered using a motivational communication style that was patient-centred and adapted to patients' levels of readiness for change [30]. Patients were randomly assigned (1/1/1/1) to 12 weeks of once-daily tiotropium $5 \mu \mathrm{g}(\mathrm{SMBM}+\mathrm{T}), \mathrm{T} / \mathrm{O} 5 / 5 \mu \mathrm{g}(\mathrm{SMBM}+\mathrm{T} / \mathrm{O}), \mathrm{T} / \mathrm{O} 5 / 5 \mu \mathrm{g}$ plus ExT $(\mathrm{SMBM}+\mathrm{T} / \mathrm{O}+\mathrm{ExT})$ or placebo $(\mathrm{SMBM}+\mathrm{P})$, all using the Respimat device after a 4 -week washout period. Patient inclusion and exclusion criteria have been previously published [26].

\section{Outcomes}

Primary and secondary outcomes of the main trial

The primary outcome of the PHYSACTO trial [28] was EET (seconds) during an endurance shuttle walk test (ESWT) to symptom limitation after 8 weeks, reflecting the duration of the ExT programme [27]. The secondary outcome was levels of daily physical activity, measured according to step counting using a triaxial accelerometer (DynaPort MoveMonitor; McRoberts, The Hague, The Netherlands). Average number of steps per day and average daily walking time were used as measures of physical activity, and gravitational acceleration $\left(1 \times g=9.81 \mathrm{~m} \cdot \mathrm{s}^{-2}\right)$ was used to measure physical activity intensity. Distance walked during the 6-min walk test (6MWT) was included as a secondary endpoint to assess walking capacity during self-paced walking. The $6 \mathrm{MWT}$ was performed $3 \mathrm{~h}$ after inhalation of study medication at weeks 8 and 12 (i.e. $1 \mathrm{~h}$ after the ESWT). These results have been published elsewhere and will not be re-reported here [28].

\section{Psychological and cognitive outcomes}

The primary outcomes of interest in this study were depression and anxiety levels, likely "caseness" of major depressive disorder, and cognitive function.

\section{Hospital Anxiety and Depression Scale}

The Hospital Anxiety and Depression Scale (HADS) is a 14-item self-report questionnaire that assesses the previous week's symptoms of depression and anxiety. The depression (HADS-D) and anxiety (HADS-A) subscales each include seven questions rated on a four-point scale from 0 to 3 (range 0-42), where scores $\geqslant 8$ are considered clinically significant $[31,32]$. The HADS has demonstrated excellent psychometric properties, including a Cronbach's $\alpha$ coefficient of 0.91 in patients with COPD.

\section{Patient-Health Questionnaire-9}

The Patient-Health Questionnaire (PHQ)-9 is a self-report questionnaire designed to assess clinical symptoms of major depressive disorder (MDD) according to Diagnostic and Statistical Manual of Mental Disorders (4th Edn) criteria. It includes nine questions rated on a scale from 0 to 3 (range 0-27), where scores $\geqslant 5$ are considered the lower limit of clinically significant MDD symptoms. The PHQ-9 has excellent psychometric properties, including sensitivity and specificity of $88 \%$ for MDD associated with scores $>10[33]$. 
Montreal Cognitive Assessment Test

The Montreal Cognitive Assessment Test (MoCA) is a rapid screening tool for mild cognitive dysfunction. It assesses various domains, including executive function, attention, concentration, language, memory, visuospatial skills, abstract thinking and orientation. The MoCA is validated for use among adults aged 55-85 years, has high test-retest reliability (with no significant learning effects when administered at 3-month intervals) and good internal consistency [34]. The maximum score on the MoCA is 30, with scores $\geqslant 26$ denoting normal cognitive functioning and scores $<26$ denoting the lower cut-off for mild cognitive impairment [34].

Questionnaires were used as a part of the behavioural modification intervention to assess cognitive function, level of depression and anxiety. They were completed at visit 1 (baseline or 4 weeks before treatment initiation), and then repeated at visits 5 (after 8 weeks of treatment and 12 weeks post-baseline) and 8 (after 12 weeks of treatment and 16 weeks post-baseline).

\section{Statistical analyses}

Baseline characteristics were compared for the four treatment groups using ANOVA or the Kruskal-Wallis test for continuous variables, and the Chi-squared test for categorical variables. To assess the changes of psychological and cognitive outcomes after 12 weeks of treatment, generalised estimating equations with a repeat statement and an unstructured correlation structure were performed, using a normal distribution for continuous outcomes with an identity link and binomial distributions for categorical outcomes with a logarithmic link. This approach accounts for the correlation of measures at different time-points. All continuous outcomes were classified as categorical variables based on minimal clinically important difference scores; models were selected based on normal distributions for continuous outcomes with an identity link and binomial distributions for categorical outcomes with a logarithmic link. Tukey adjustment for multiple comparisons were also performed for significant overall p-values. Mixed-effects linear models were used to estimate the relationship between physical activity, exercise capacity, and psychological and cognitive outcomes, with random intercepts to capture individual-specific change, adjusted for covariates. All analyses were performed using SAS, version 9.4 (SAS Institute, Cary, NC, USA); p-values $<0.05$ were considered statistically significant.

\section{Results}

\section{Sample characteristics}

Baseline characteristics were generally balanced between groups (table 1). Patients were $66 \%$ male and mean \pm sD age was $65 \pm 6.6$ years. $37 \%$ were current smokers with a history of $46.9 \pm 23.8$ pack-years and a mean body mass index (BMI) of $29.0 \pm 21.3 \mathrm{~kg} \cdot \mathrm{m}^{-2}$. A total of $55.6 \%$ were at Global Initiative for Chronic Obstructive Lung Disease stage 3 or 4 and mean forced expiratory volume in $1 \mathrm{~s}\left(\mathrm{FEV}_{1}\right)$ was $48.7 \pm 13.2 \%$ predicted. Baseline HADS-A and HADS-D scores were 4.8 \pm 3.2 and 3.8 \pm 2.7 , respectively, and MDD scores (PHQ-9) were 4.6 \pm 3.8 , denoting low anxiety and depression levels in this cohort. Cognitive function (MoCA) scores were, on average, 25.6 \pm 3.1 , which is just within the range of mild cognitive impairment (table 1).

\section{Changes in psychological and cognitive outcomes after 12 weeks}

There were no significant between-group differences in any outcome with the exception of cognitive function, where significantly greater increases in cognitive function (MoCA total scores) were observed between the SMBM+T and SMBM+T/O treatment groups compared with the SMBM+P group (table 2). As a result, we conducted all further analyses on the sample as a cohort, adjusting for treatment group.

Independent of treatment group and other covariates, HADS-A, HADS-D and PHQ-9 scores all decreased significantly from baseline to post-treatment $(\mathrm{p}<0.001)$ (table 2 and figure 2$)$. However, average baseline levels of psychological distress were quite low (all in the normal range) (table 2). Therefore, changes in the proportion of patients exhibiting clinically significant levels of anxiety (HADS-A $\geqslant 8$ ), depression (HADS-D $\geqslant 8$ ) and MDD symptoms (PHQ-9 $\geqslant 5$ ) were also analysed. Treatment was associated with a significant reduction in the proportion of patients exhibiting clinically significant anxiety and MDD $(\mathrm{p}<0.001)$ (figure 3). A similar pattern was observed for cognitive function, where scores on the MoCA increased significantly (denoting improved cognitive function) from pre- to post-treatment (table 2 and figure 3). Like with the psychological outcomes, we found that that the proportion of patients scoring within the range of mild cognitive impairment (MoCA <26) improved significantly from pre- $(44 \%)$ to post-treatment $(19 \%)(\mathrm{p}<0.001)$ (figure 2$)$.

\section{Association between increases in physical activity and exercise capacity and changes in} psychological and cognitive outcomes

Independent of treatment group and other covariates, we observed a significant association between increases in all physical activity variables and all but one exercise capacity variable (ESWT walk duration) 
TABLE 1 Baseline characteristics of the eligible subjects by self-management behaviour modification (SMBM) group (n=304)

Variables

\section{Subjects $\mathbf{n}$}

Demographics

Age years

Males

$\mathrm{BMI} \mathrm{kg} \cdot \mathrm{m}^{2}$

Ever-smokers

Current smokers

Smoking history pack-years

Symptoms and clinical data

mMRC dyspnoea score

Chronic bronchitis

Sputum-producing cough

Emphysema

SGRQ total score

MoCA total score

HADS-A score

HADS-D score

PHQ-9 total score

Lung function

$\mathrm{FEV}_{1} \mathrm{~L}$

$\mathrm{FEV}_{1} \%$ predicted

$\mathrm{FEV}_{1} /$ FVC \%

COPD GOLD stages 1-2

COPD GOLD stages 3-4

\section{Exercise function}

ESWT

Walk distance $m$

Walk duration min

Shuttles

Walk distance in 6MWT m

Activity monitor/pedometer

Steps per day

Walking duration min

Walking intensity $\times g$

Total

304

$64.8 \pm 6.6$

$201(66.1 \%)$

$29.0 \pm 21.3$

$192(63.2 \%)$

$112(36.8 \%)$

$46.9 \pm 23.8$

$1.3 \pm 0.9$

$143(47.0 \%)$

$184(60.5 \%)$

$140(46.1 \%)$

$40.1 \pm 15.7$

$25.6 \pm 3.1$

$4.8 \pm 3.2$

$3.8 \pm 2.7$

$4.6 \pm 3.8$

$1.4 \pm 0.5$

$48.7 \pm 13.2$

$47.0 \pm 10.5$

$129(42.4 \%)$

169 (55.6\%)

$$
\begin{gathered}
404.6 \pm 290.1 \\
4.9 \pm 3.3 \\
40.5 \pm 29.0 \\
448.2 \pm 101.2
\end{gathered}
$$
$5423.1 \pm 2820.2$
$67.0 \pm 32.3$
$0.2 \pm 0.0$

T/O FDC $(5 / 5 \mu \mathrm{g})+$ SMBM+ExT T/O FDC $(5 / 5 \mu \mathrm{g})+$ SMBM

76

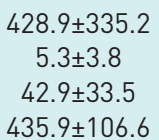
$5590.2 \pm 2683.4$
$68.4 \pm 30.4$
$0.2 \pm 0.0$

76

$64.9 \pm 6.9$

$48(63.2 \%)$

$27.3 \pm 5.0$

47 (61.8\%)

29 (38.2\%)

$41.9 \pm 19.6$

$1.3 \pm 0.9$

$39(51.3 \%)$

43 (56.6\%)

33 (43.4\%)

$40.6 \pm 17.1$

$25.3 \pm 3.4$

$4.6 \pm 3.2$

$3.3 \pm 2.4$

$4.2 \pm 3.4$

$1.4 \pm 0.4$

$49.6 \pm 12.5$

$48.7 \pm 11.8$

$34(44.7 \%)$

38 (50.0\%)

$436.8 \pm 300.2$

$5.2 \pm 3.4$

$43.7 \pm 30.0$

$458.0 \pm 100.4$

$\mathrm{T}(5 \mu \mathrm{g})+\mathrm{SMBM}$

76

76

$65.1 \pm 6.4$

$55(72.4 \%)$

$32.1 \pm 41.8$

$49(64.5 \%)$

27 (35.5\%)

$51.0 \pm 28.0$

$64.4 \pm 6.6$

$53(69.7 \%)$

$28.3 \pm 4.7$

$48(63.2 \%)$

28 (36.8\%)

$48.6 \pm 22.0$

$1.3 \pm 0.8$

$34(44.7 \%)$

$44(57.9 \%)$

$33(43.4 \%)$

$39.1 \pm 15.3$

$25.4 \pm 3.0$

$5.2 \pm 3.2$

$4.2 \pm 2.8$

$5.0 \pm 4.0$

$1.4 \pm 0.8$

30 (39.5\%)

$48(63.2 \%)$

$36(47.4 \%)$

$41.7 \pm 14.5$

$25.8 \pm 3.1$

$4.9 \pm 3.2$

$4.1 \pm 2.4$

$5.0 \pm 3.8$

$1.4 \pm 0.4$

$1.4 \pm 0.5$

$48.8 \pm 13.9$

$47.2 \pm 13.3$

$46.8 \pm 9.9$

$45.9 \pm 10.3$

30 (39.5)

$30(39.5 \%)$

45 (59.2)

$46(60.5 \%)$

$388.9 \pm 265.4$
$4.8 \pm 3.0$
$38.9 \pm 26.5$
$450.2 \pm 94.7$

$363.8 \pm 252.0$

$4.5 \pm 3.0$

$36.4 \pm 25.2$

$448.7 \pm 103.6$
$5723.2 \pm 3065.9$
$69.9 \pm 36.3$
$0.2 \pm 0.0$

$5223.7 \pm 2769.6$

$65.3 \pm 31.7$

$0.2 \pm 0.0$

Data are presented as mean \pm SD unless otherwise stated. $p$-values were obtained by performing ANOVA or Kruskal-Wallis test for continuous variable and Chi-Square test for category variables. T: tiotropium; 0: olodaterol; FDC: fixed-dose combination; ExT: exercise training; BMI: body mass index; mMRC: modified Medical Research Council; SGRQ: St George's Respiratory Questionnaire; MoCA: Montreal Cognitive Assessment; HADS: Hospital Anxiety and Depression Scale; A: anxiety; D: depression; PHQ: Patient-Health Questionnaire; FEV ${ }_{1}$ : forced expiratory volume in $1 \mathrm{~s}$; FVC: forced vital capacity; GOLD: Global Initiative for Chronic Obstructive Lung Disease; ESWT: endurance shuttle walk test; 6MWT: 6-min walk test.

and improvements (decreases) in depression scores (HADS-D and PHQ-9) after adjustment for covariates (table 3). Every 100-step increase per day was associated with a 0.009-point reduction in the HADS-D $(\mathrm{p}=0.003)$ and a 0.01 -point reduction in the PHQ-9 $(\mathrm{p}=0.012)$. Every 10 -min increase in walk duration was associated with a 0.08-point reduction in the HADS-D ( $p=0.003)$ and a 0.08 -point reduction in the PHQ-9 $(\mathrm{p}=0.033)$. Every 1-point increase in walking intensity was associated with a 14.94-point reduction in the HADS-D $(\mathrm{p}<0.0001)$ and a 10.31-point reduction in the PHQ-9 $(\mathrm{p}=0.032)$. Similar to the physical activity variables, every $10-\mathrm{m}$ increase in ESWT distance was associated with a 0.007 -point reduction in the HADS-D ( $p=0.005)$ and 0.007-point reduction in PHQ-9 $(\mathrm{p}=0.049)$; every 1-min increase in ESWT duration was associated with a 0.05 -point reduction in the HADS-D ( $p=0.016)$; every 5-point increase in ESWT number was associated with a 0.04-point reduction in the HADS-D ( $p=0.004)$ and 0.03-point reduction in the PHQ-9 ( $\mathrm{p}=0.046)$; and every $10-\mathrm{m}$ increase in 6MWT distance was associated with a 0.04-point reduction in the HADS-D $(\mathrm{p}<0.001)$ and a 0.05-point reduction in the PHQ-9 $(\mathrm{p}=0.001)$.

Associations between physical activity, exercise capacity changes, and improvements in anxiety and cognitive function were less consistent. HADS-A scores decreased by 10.94 points in association with every 1-point increase in daily walking intensity $(\mathrm{p}=0.013)$, and MoCA scores increased by 0.04 with every $10-\mathrm{m}$ increase in distanced walked during the $6 \mathrm{MWT}(\mathrm{p}=0.005)$. 
TABLE 2 The changes in psychological and cognitive outcomes after 12 weeks by self-management behaviour modification (SMBM) group

\begin{tabular}{|c|c|c|c|c|c|c|c|c|c|c|c|}
\hline \multirow[t]{2}{*}{ Variables } & \multicolumn{2}{|c|}{ Total $(n=262)$} & \multicolumn{2}{|c|}{$\begin{array}{l}\text { T/0 FDC }(5 / 5 \mu \mathrm{g}) \\
+ \text { SMBM+EXT }(n=65)\end{array}$} & \multicolumn{2}{|c|}{$\begin{array}{c}\text { T/O FDC }(5 / 5 \mu \mathrm{g})+\text { SMBM } \\
(\mathrm{n}=69)\end{array}$} & \multicolumn{2}{|c|}{$\mathrm{T}(5 \mu \mathrm{g})+\mathrm{SMBM}(\mathrm{n}=64)$} & \multicolumn{2}{|c|}{ Placebo+SMBM (n=64) } & \multirow[t]{2}{*}{$\begin{array}{c}\text { Four-group } \\
\text { comparison } p \text {-value }\end{array}$} \\
\hline & Baseline & Week 12 & Baseline & Week 12 & Baseline & Week 12 & Baseline & Week 12 & Baseline & Week 12 & \\
\hline MoCA total score ${ }^{\#}$ & $25.5 \pm 3.2$ & $27.5 \pm 2.6^{* * *}$ & $25.7 \pm 3.0$ & $27.7 \pm 2.5^{* * *}$ & $25.3 \pm 3.5$ & $27.5 \pm 2.6 * * *, 9$ & $25.2 \pm 3.1$ & $27.4 \pm 2.5^{* * *,+}$ & $25.8 \pm 3.2$ & $27.2 \pm 2.7^{* * *, 9},+$ & 0.043 \\
\hline MoCA $<26^{\#}$ & $116(44.3 \%)$ & $49(19.0 \%)^{* * *}$ & $26(40.0 \%)$ & $11(16.9 \%)^{* * *}$ & $34(49.3 \%)$ & $11(15.9 \%)^{* * *}$ & $31(48.4 \%)$ & $13(20.6 \%)^{* * *}$ & $25(39.1 \%)$ & $14(23.0 \%)^{* *}$ & 0.106 \\
\hline HADS-A score & $4.9 \pm 3.3$ & $3.8 \pm 3.0 * * *$ & $4.9 \pm 3.4$ & $3.8 \pm 2.5^{* *}$ & $4.5 \pm 3.2$ & $3.1 \pm 3.2^{* * *}$ & $5.0 \pm 3.3$ & $3.9 \pm 3.0 * * *$ & $5.0 \pm 3.3$ & $4.3 \pm 3.3$ & 0.225 \\
\hline HADS-A $\geqslant 8$ & $51(19.5 \%)$ & $31(12.0 \%)^{* *}$ & $10(15.4 \%)$ & $6(9.2 \%)$ & $11(15.9 \%)$ & 10 (14.5\%) & $17(26.6 \%)$ & $8(12.7 \%)^{* *}$ & $13(20.3 \%)$ & $7(11.5 \%)$ & 0.676 \\
\hline HADS-D score & $3.8 \pm 2.6$ & $3.1 \pm 2.6 * * *$ & $3.8 \pm 3.1$ & $2.6 \pm 2.3^{* * *}$ & $3.2 \pm 2.3$ & $2.6 \pm 2.4^{*}$ & $3.8 \pm 2.7$ & $3.4 \pm 2.9$ & $4.2 \pm 2.3$ & $3.6 \pm 2.8$ & 0.288 \\
\hline HADS-D $\geqslant 8$ & $24(9.2 \%)$ & $20(7.8 \%)$ & $7(10.8 \%)$ & $3(4.6 \%)$ & $3(4.3 \%)$ & $4(5.8 \%)$ & $8(12.5 \%)$ & $7(11.1 \%)$ & $6(9.4 \%)$ & $6(9.8 \%)$ & 0.269 \\
\hline PHQ-9 total score & $4.5 \pm 3.7$ & $3.1 \pm 3.3^{* * *}$ & $4.4 \pm 4.0$ & $2.7 \pm 2.8 * * *$ & $3.9 \pm 3.1$ & $2.7 \pm 3.0^{* * *}$ & $4.4 \pm 3.6$ & $3.1 \pm 3.2^{* * *}$ & $5.1 \pm 4.0$ & $3.8 \pm 3.9 *$ & 0.253 \\
\hline$P H Q-9 \geqslant 5$ & $98(37.4 \%)$ & $62(24.0 \%)^{* * *}$ & $27(41.5 \%)$ & $12(18.5 \%)^{* * *}$ & $22(31.9 \%)$ & $13(18.8 \%)^{* *}$ & $24(37.5 \%)$ & $16(25.4 \%)$ & $25(39.1 \%)$ & $21(34.4 \%)$ & 0.282 \\
\hline
\end{tabular}

Data are presented as mean \pm SD unless otherwise stated. p-values were obtained by performing a generalised estimating equation (GEE) model with type III likelihood ratio statistics via normal distribution with a logarithmic link function for continuous variables, and binary distribution with a logit link function for category variables. For the four-group comparison, we used a GEE model with repeated statement to test the timexgroup interaction term and estimated whether the average change in the outcome from baseline to week 12 differed in the two groups. T: tiotropium; 0: olodaterol; FDC: fixed-dose combination; ExT: exercise training; MoCA: Montreal Cognitive Assessment; HADS: Hospital Anxiety and Depression Scale; A: anxiety; D: depression; PHQ: Patient-Health Questionnaire. ": means and proportions adjusted for correlation between repeated measures. *: $\mathrm{p}<0.05$; ${ }^{* *}$ : $\mathrm{p}<0.01$; ***: $\mathrm{p}<0.001$, comparing baseline and week 12 after Tukey adjustment. " and ${ }^{+}$: means with same symbol are significantly different from each other in terms of the average change in the outcome from baseline to week $12(p<0.05)$. 

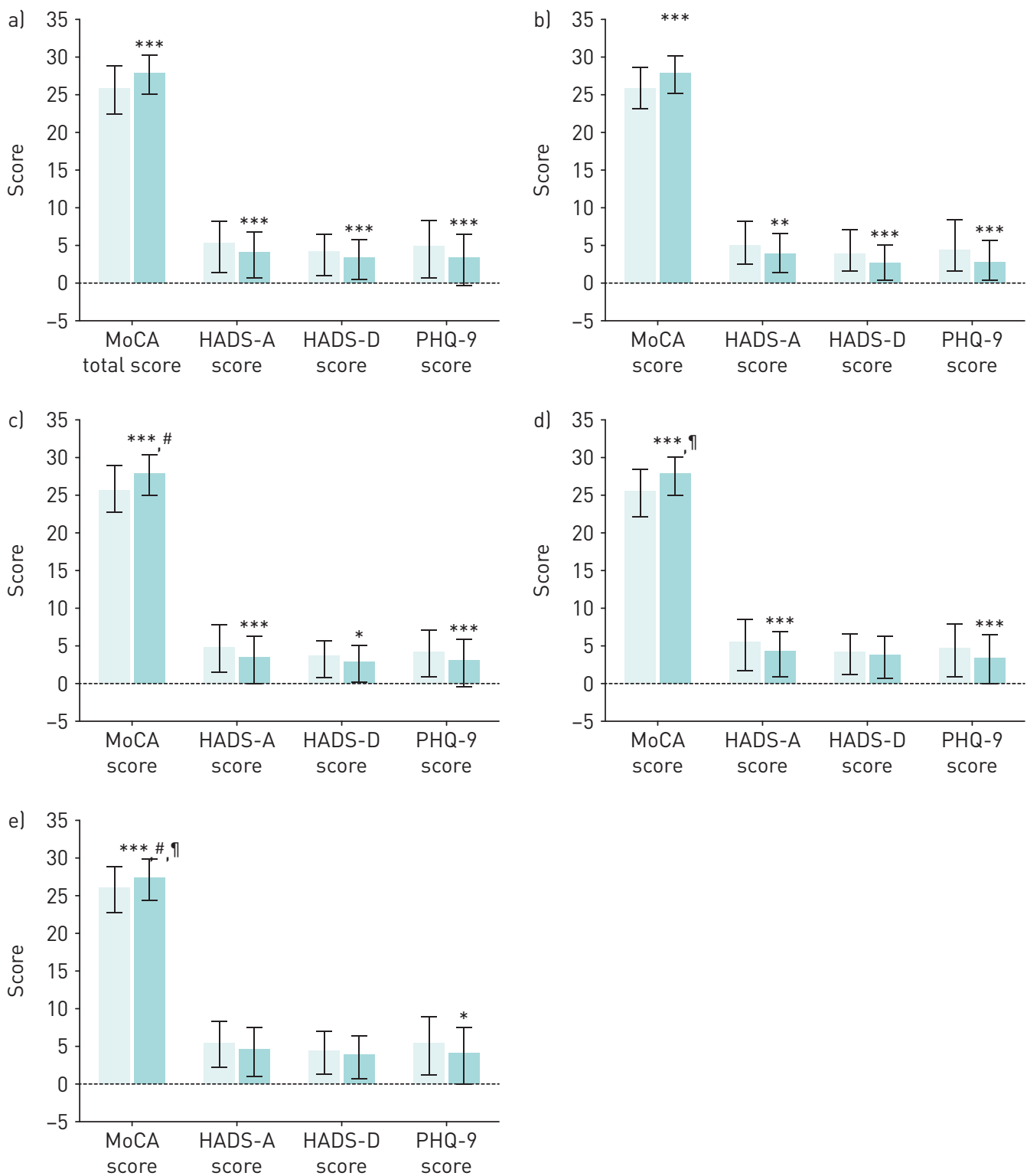

FIGURE 2 Changes in psychological and cognitive outcomes after 12 weeks of with tiotropium (T), T/olodaterol (0) or placebo. a) Total; b) self-management behaviour modification (SMBM)+T/O+exercise training; c) SMBM+T/O; d) SMBM+T; el SMBM+placebo. MoCA: Montreal Cognitive Assessment; HADS: Hospital Anxiety and Depression Scale; A: anxiety; D; depression; PHQ: Patient-Health Questionnaire. *: $p<0.05$; **: $p<0.01$; ${ }^{* * *}$ : $p<0.001$, by comparing baseline and week 12 after Tukey adjustment; ${ }^{\#}$ : $p<0.05$, SMBM+T/0 versus SMBM+placebo; ${ }^{\Uparrow}:$ p $<0.05$ SMBM+T versus SMBM+placebo.

\section{Discussion}

This study assessed the effects of a SMBM programme alone or in combination with bronchodilator therapy (tiotropium or T/O), with or without ExT, on psychological and cognitive outcomes in patients with COPD in a secondary analysis of data from the PHYSACTO trial. The only between-group differences were observed for cognitive function, where greater increases in MoCA total scores were observed in the SMBM+T and SMBM+T/O groups (but not the SMBM+T/O+ExT group) compared with the $\mathrm{SMBM}+\mathrm{P}$ group. Contrary to our hypotheses, this suggests that being exposed to ExT does not seem to afford any additional benefit for cognitive function over and above SMBM (which emphasised increasing daily levels of physical activity)+bronchodilator therapy alone. This contributed to treating the sample as a cohort and conducting all further analyses adjusting for treatment group. These analyses revealed that levels of anxiety, depression, MDD symptoms and cognitive function all showed statistically and clinically significant improvements from pre- to post-treatment, irrespective of treatment group, which is consistent with previous reports $[35,36]$. 
TABLE 3 The association between psychological and cognitive outcomes and physical activity (PA) and exercise capacity variables using multiple mixed effect random models

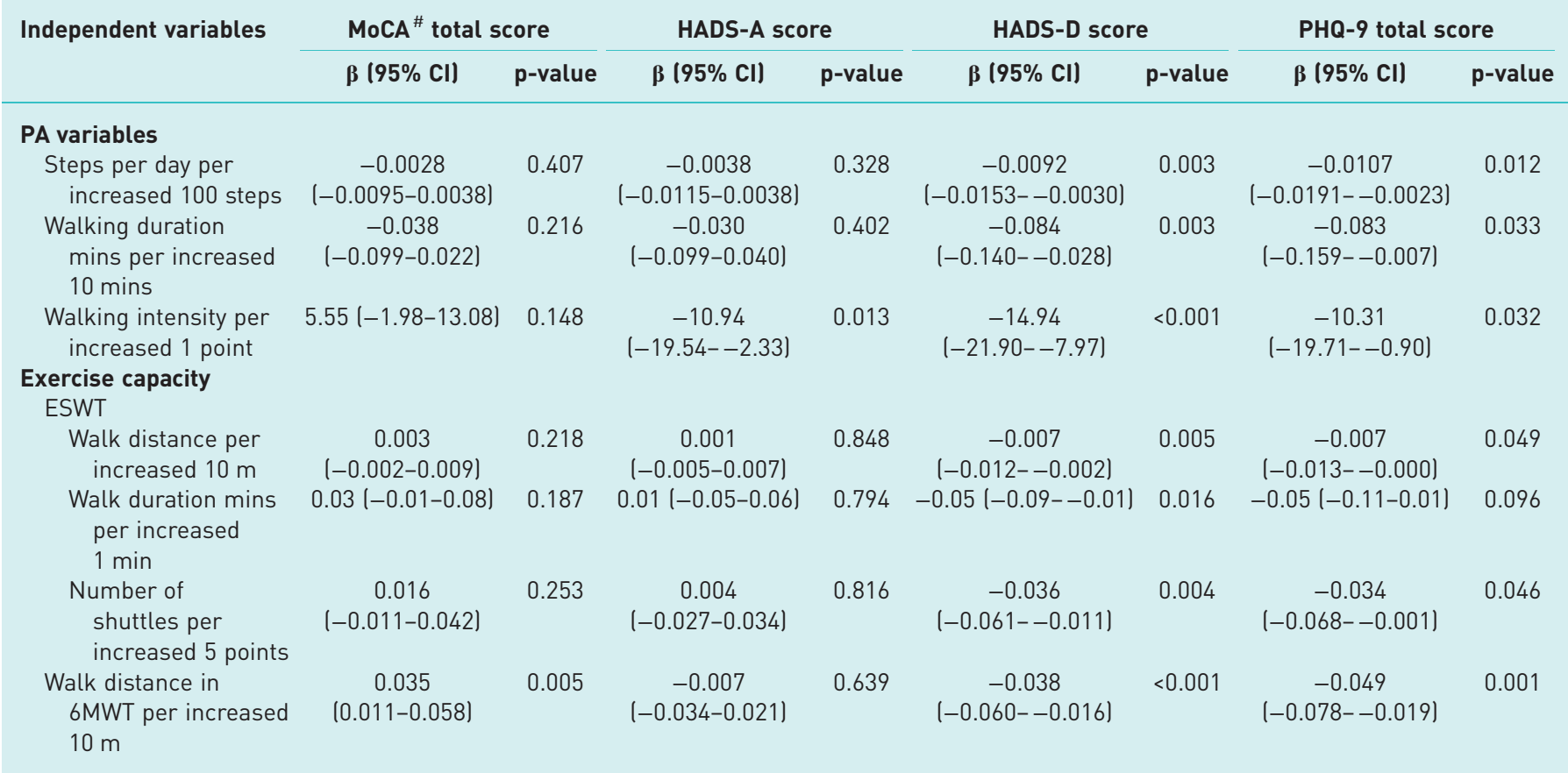

Models adjusted for baseline age, sex, body mass index, smoking pack-years, \% predicted forced expiratory volume in $1 \mathrm{~s}$, treatment group and time variable week. MoCA: Montreal Cognitive Assessment; HADS: Hospital Anxiety and Depression Scale; A: anxiety; D: depression; PHQ: Patient-Health Questionnaire; ESWT: endurance shuttle walk test; 6MWT: 6-min walk test." : adjusted for correlation between repeated measures.

Anxiety improved with greater increases in physical activity, cognitive function improved with greater increases in exercise capacity, and depression improved with greater increases in both physical activity and exercise capacity. Findings of improved cognitive function associated with increased exercise capacity are consistent with previous exercise intervention studies in patients with chronic disease [22] and patients with COPD $[25,36]$. Results linking increased exercise capacity to improvements in depression are also consistent with previous reports, where studies have reported improvements in depression following pulmonary rehabilitation programmes [37]. Depression also improved with increases in physical activity, as did anxiety, in line with previous reports linking physical activity to improvements in depression and anxiety $[38,39]$.

Although this study was not designed to explore the mechanisms responsible for the positive associations between increases in physical activity/exercise capacity and differential improvements in psychological and cognitive outcomes, it is possible that only patients who were able to achieve certain thresholds of physiological arousal (i.e. enough to improve exercise capacity) experienced improvements in cognitive function with increased exercise capacity. Previous research has shown that engaging in at least 45-60 min of moderate-intensity aerobic exercise about three times per week [40] affects the aspects of brain structure and function associated with cognition, including increasing cerebral blood flow [41] and stimulating central neurotransmitter release [42]. Cognitive function did not seem to improve with increasing physical activity, suggesting that being physically active alone may not produce sufficient physiological arousal to impact the biological processes associated with cognitive performance. This is consistent with the results of at least one prospective study that showed that increasing levels of physical activity among patients with COPD undergoing pulmonary rehabilitation did not translate into increases in exercise capacity [38].

Conversely, increases in physical activity, and not exercise capacity, were associated with improvements in anxiety. Highly anxious patients typically avoid engaging in situations or behaviours that they believe will cause psychological or physical discomfort [43]. Patients with COPD who have high anxiety levels have been shown to avoid social situations due to fears of being embarrassed by their symptoms, or of engaging in physical activity for fear of exacerbating symptoms or experiencing pain [44]. It is therefore possible that patients who successfully increase their daily level of physical activity learn through gradual exposure that increasing physical activity is safe and achievable, thereby reducing overall anxiety levels. 

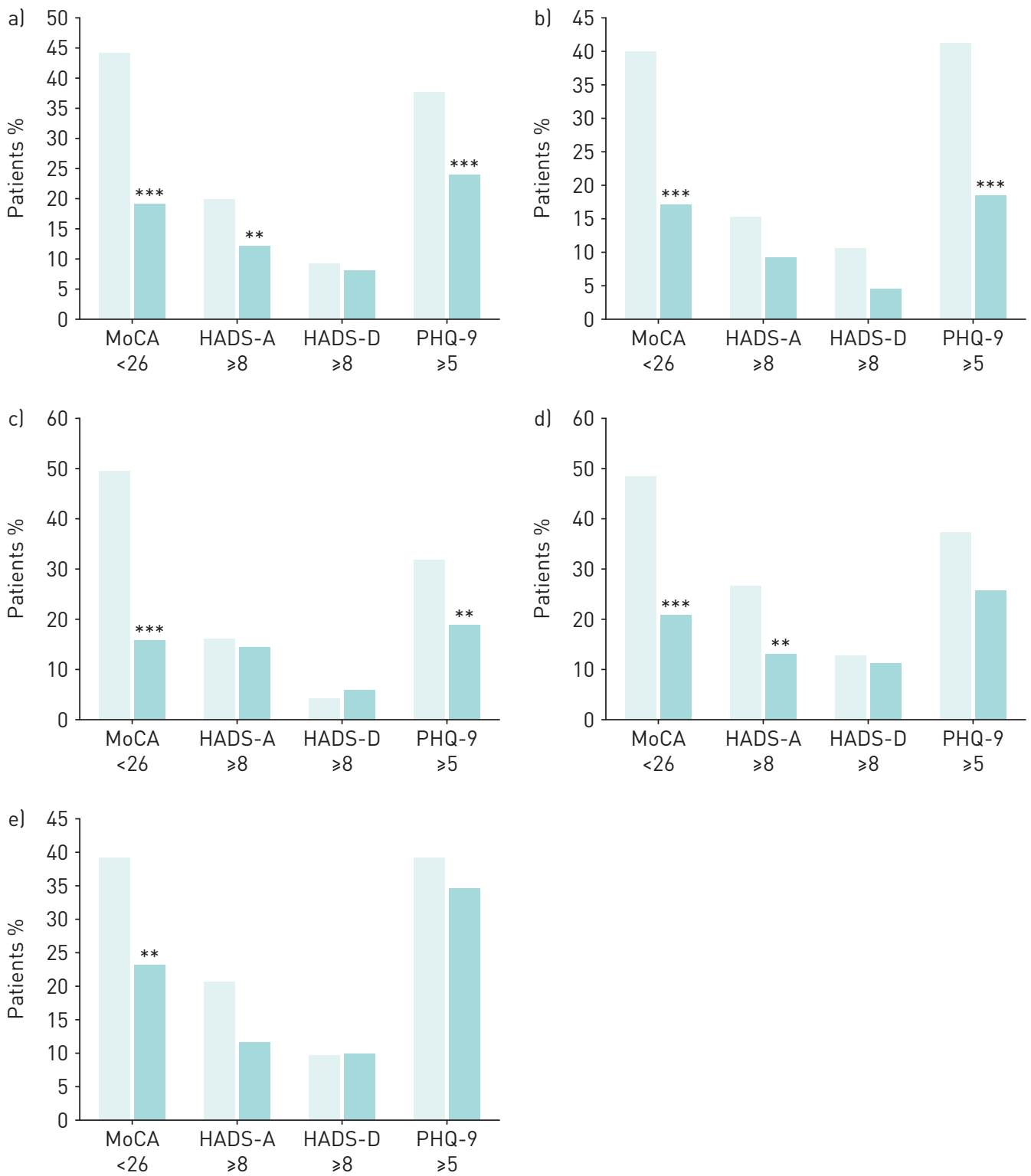

FIGURE 3 Proportion of patients experiencing changes in psychological and cognitive outcomes after 12 weeks of treatment with tiotropium (T), T/olodaterol (0) or placebo. a) Total; b) self-management behaviour modification (SMBM)+T/O+exercise training; c) SMBM+T/O; d) SMBM+T; el SMBM+placebo. MoCA: Montreal Cognitive Assessment; HADS: Hospital Anxiety and Depression Scale; A: anxiety; D; depression; PHQ: Patient-Health Questionnaire. ${ }^{* *}: p<0.01 ;{ }^{* * *}: p<0.001$.

Depressive symptoms and MDD scores improved as a result of both increases in physical activity and increases in exercise capacity. This finding suggests that depression/MDD may improve through both biological and behavioural pathways, which is consistent with the major theories and treatments of depression [45]. Depression is thought to occur as a result of several neurophysiological factors, including hormonal and neurotransmitter imbalances causing dysregulations in emotional processing and behavioural activation [46]. Like cognitive function, it is possible that achieving the thresholds of physiological arousal sufficient to improve exercise capacity can result in improvements in depression/ MDD. However, the fact that physical activity also improved depression/MDD scores is consistent with behavioural theories of depression that link depression to maladaptive thoughts and behaviours [47]. Unlike exercise capacity, physical activity is a behaviour that tends be self-directed and motivated by psychological factors such as beliefs, self-efficacy and outcome expectancies [48]. The behavioural activation required to engage in higher levels of physical activity may have been sufficient to change beliefs and improve self-efficacy, which may have in turn resulted in improvements in depressed mood. 
This study has some limitations. First, the results should be interpreted with caution as this was a secondary analysis of data from a study with another primary objective, so was not specifically powered for these outcomes. Second, a treatment-free control group was not included, therefore the extent to which psychological and cognitive outcomes may have improved spontaneously cannot be ruled out. However, previous research has shown that cognitive function among older adults tends to be degenerative and rarely improves outside of treatment [49]. While depression and anxiety levels can vary over time independently of formal intervention, it is less common to see such a consistent pattern of clinically significant changes outside of treatment [50]. Our psychological outcomes were self-reported, not objectively assessed (i.e. using a psychiatric interview) and may be subject to self-report bias. Further, baseline levels of anxiety and depression on the HADS and PHQ-9 were extremely low, suggesting that a possible regression to the mean effect could have been observed post-intervention. However, we did use valid and reliable self-reported questionnaires that have established clinical cut-offs. This allows for the classification of clinically significant levels of psychological distress which we were able to show shifted in a clinically meaningful way post-treatment. The 12-week duration of the study was also very short and may have limited our ability to detect larger effects on outcomes (e.g. cognitive function) that may take more time to change. However, it is noteworthy that despite the short intervention period, we still observed both statistically and clinically significant improvements on depression, anxiety and cognitive function. Lastly, the MoCA may be subject to some practice effects. However, data suggest there are little-to-no practice effects when the MoCA is administered at intervals $\geqslant 3$ months, which is less than the pre- to post-treatment assessment interval in the present study. To account for this, we adjusted for repeated assessments, and even when practice effects are observed, they tend to account for $<10 \%$ of scores [34]

Despite some limitations, this study has several notable strengths. It includes a large sample of well-characterised patients with COPD from 12 countries, which increases the generalisability of the results. The interventions (including SMBM) were carefully implemented: all case managers received standardised training on the protocol and its delivery, and underwent multiple fidelity assessments (i.e. self-assessments of performance, and expert-rater assessments of audio-taped individual and group sessions). Only valid and reliable psychological and cognitive measures were used, and the administering personnel, who were blind to treatment group, received standardised training and ongoing supervision. Physical activity was assessed objectively using accelerometers, and all results were adjusted for important covariates, including age, sex, BMI, smoking pack-years, pulmonary function $\left(\mathrm{FEV}_{1} \%\right.$ predicted) and baseline levels of each outcome variable, attesting to the robustness of the findings.

In summary, our data indicate that SMBM aimed at improving daily physical activity with or without bronchodilators (tiotropium or T/O), with or without ExT, was associated with both statistically and clinically significant improvements in psychological cognitive functioning in moderate-severe COPD patients in the PHYSACTO study. However, anxiety improved with greater increases in physical activity, cognitive function improved with greater increases in exercise capacity, and depression improved with increases in either physical activity or exercise capacity. Although this was a secondary analysis of data from a study with another primary objective, the overall pattern of results indicates that interventions that increase daily physical activity and/or exercise capacity may be beneficial for improving psychological and cognitive outcomes in patients with COPD and provides additional evidence of the importance of increasing access to physical activity/exercise-based interventions in patients with COPD.

Acknowledgements: Special thanks to the study participants and staff in 11 countries. Medical writing assistance was supported financially by Boehringer Ingelheim and provided by Martina Stagno d'Alcontres at MediTech Media (Manchester, UK). The authors meet the criteria for authorship as recommended by the International Committee of Medical Journal Editors.

PHYSACTO Behaviour Modification Team: J. Bourbeau (McGill University Health Center, Montreal, QC, Canada); K.L. Lavoie (University of Quebec at Montreal, Montreal, QC, Canada); M. Sedeno (McGill University Health Center, Montreal, QC, Canada); D. De Sousa (Boehringer Ingelheim, Burlington, ON, Canada); A. Hamilton (Boehringer Ingelheim, Burlington, ON, Canada). PHYSACTO Study Team: T. Troosters (University Hospital Leuven, Leuven, Belgium); D. Erzen (Boehringer Ingelheim, Biberach an der Riss, Germany); L. Korducki; (Boehringer Ingelheim, Ridgefield, CT, USA), N. Leidy (Evidera, Bethesda, MD, USA) W. Janssens (University Hospital Gasthuisberg, Leuven, Belgium).

Support statement: The PHYSACTO trial (NCT02085161) was supported by Boehringer Ingelheim International $\mathrm{GmbH}$. K.L. Lavoie received salary support from a Senior Investigator Award from the Fonds de la recherche du Québec - Santé. Funding information for this article has been deposited with the Crossref Funder Registry.

Conflict of interest: K.L. Lavoie reports investigator-initiated research support and speaking fees from Abbvie, consulting fees and speaking fees from Boehringer Ingelheim, Astellas and Novartis, consulting fees from Janssen and AstraZeneca, and speaking fees from Bayer and Mundipharma, outside the submitted work. M. Sedeno is an employee of RESPIPLUS and, in this salaried position, works with a variety of companies and receives no payment or honoraria directly from 
them for services rendered. A. Hamilton is an employee of Boehringer Ingelheim (Canada) Ltd. P-Z. Li has nothing to disclose. D. De Sousa is an employee of Boehringer Ingelheim. T. Troosters reports that his institute received speakers' fees and consultancy fees for activities related to physical activity and exercise in COPD from Boehringer Ingelheim, and for activities around physical activity and exercise from AstraZeneca; and grants from PROactive, during the conduct of the study; and grants from Research Foundation Flanders, outside the submitted work. F. Maltais reports personal fees and nonfinancial support from Boehringer Ingelheim, GlaxoSmithKline, AstraZeneca, Novartis, and Griffols, outside the submitted work. J. Bourbeau reports grants from McGill University, the Research Institute of the McGill University Health Centre, GlaxoSmithKline, Aerocrine, the Canadian Respiratory Research Network-Canadian Institute of Health Research, Almirall, AstraZeneca, Boehringer Ingelheim and Novartis, outside the submitted work.

\section{References}

1 O’Donnell DE, Laveneziana P. Physiology and consequences of lung hyperinflation in COPD. Eur Respir J 2006; 15: 61-67.

2 O'Donnell DE, Casaburi R, Frith $\mathrm{P}$, et al. Effects of combined tiotropium/olodaterol on inspiratory capacity and exercise endurance in COPD. Eur Respir J 2017; 49: 1601348.

3 Ramon MA, Ter Riet G, Carsin AE, et al. The dyspnoea-inactivity vicious circle in COPD: development and external validation of a conceptual model. Eur Respir J 2018; 52: 1800079.

4 Neder JA, Berton DC, Muller PT, et al. Canadian Respiratory Research Network. Ventilatory inefficiency and exertional dyspnea in early chronic obstructive pulmonary disease. Ann Am Thorac Soc 2017; 14: S22-S29.

5 Global Initiative for Chronic Obstructive Lung Disease. Global Strategy for the Diagnosis, Management, and Prevention of Chronic Obstructive Pulmonary Disease: 2018 Report. https:/goldcopd.org/gold-reports/. Date last accessed: July 17, 2018.

6 Teixeira PJ, Porto L, Kristensen $\mathrm{CH}$, et al. Post-traumatic stress symptoms and exacerbations in COPD patients. COPD 2015; 12: 90-95.

7 Lu Y, Nyunt MS, Gwee X, et al. Life event stress and chronic obstructive pulmonary disease (COPD): associations with mental well-being and quality of life in a population-based study. BMJ Open 2012; 2: e001674.

8 Laurin C, Moullec G, Bacon SL, et al. Impact of anxiety and depression on chronic obstructive pulmonary disease exacerbation risk. Am J Respir Crit Care Med 2012; 185: 918-923.

9 Heerema-Poelman A, Stuive I, Wempe JB. Adherence to a maintenance exercise program 1 year after pulmonary rehabilitation: what are the predictors of dropout? J Cardiopulm Rehabil Prev 2013; 33: 419-426.

10 Giardino ND, Curtis JL, Andrei AC, et al. Anxiety is associated with diminished exercise performance and quality of life in severe emphysema: a cross-sectional study. Respir Res 2010; 11: 29.

11 Martinez $\mathrm{CH}$, Richardson CR, Han MK, et al. Chronic obstructive pulmonary disease, cognitive impairment, and development of disability: the health and retirement study. Ann Am Thorac Soc 2014; 11: 1362-1370.

12 Villeneuve S, Pepin V, Rahayel S, et al. Mild cognitive impairment in moderate to severe COPD: a preliminary study. Chest 2012; 142: 1516-1523.

$13 \mathrm{Li} \mathrm{J}$, Huang Y, Fei GH. The evaluation of cognitive impairment and relevant factors in patients with chronic obstructive pulmonary disease. Respiration 2013; 85: 98-105.

14 Cleutjens FA, Janssen DJ, Ponds RW, et al. COgnitive-Pulmonary Disease. Biomed Res Int 2014; 2014: 697825.

15 Ouellette DR, Lavoie KL. Recognition, diagnosis, and treatment of cognitive and psychiatric disorders in patients with COPD. Int J Chron Obstruct Pulmon Dis 2017; 12: 639-650.

16 Liesker JJ, Postma DS, Beukema RJ, et al. Cognitive performance in patients with COPD. Respir Med 2004; 98 : 351-356.

17 Cleutjens FAHM, Spruit MA, Ponds RWHM, et al. The impact of cognitive impairment on efficacy of pulmonary rehabilitation in patients with COPD. J Am Med Dir Assoc 2017; 18: 420-426.

18 Baird C, Lovell J, Johnson M, et al. The impact of cognitive impairment on self-management in chronic obstructive pulmonary disease: a systematic review. Respir Med 2017; 129: 130-139.

19 Hearing CM, Chang WC, Szuhany KL, et al. Physical exercise for treatment of mood disorders: a critical review. Curr Behav Neurosci Rep 2016; 3: 350-359.

20 Herring MP, Puetz TW, O'Connor PJ, et al. Effect of exercise training on depressive symptoms among patients with a chronic illness: a systematic review and meta-analysis of randomized controlled trials. Arch Intern Med 2012; 172: 101-111.

21 Sofi F, Valecchi D, Bacci D, et al. Physical activity and risk of cognitive decline: a meta-analysis of prospective studies. J Intern Med 2011; 269: 107-117.

22 Cai H, Li G, Hua S, et al. Effect of exercise on cognitive function in chronic disease patients: a meta-analysis and systematic review of randomized controlled trials. Clin Interv Aging 2017; 12: 773-783.

23 Zheng G, Xia R, Zhou W, et al. Aerobic exercise ameliorates cognitive function in older adults with mild cognitive impairment: a systematic review and meta-analysis of randomised controlled trials. Br J Sports Med 2016; 50: $1443-1450$.

24 Groot C, Hooghiemstra AM, Raijmakers PG, et al. The effect of physical activity on cognitive function in patients with dementia: a meta-analysis of randomized control trials. Ageing Res Rev 2016; 25: 13-23.

25 Aquino G, Iuliano E, di Cagno A, et al. Effects of combined training vs aerobic training on cognitive functions in COPD: a randomized controlled trial. Int J Chron Obstruct Pulmon Dis 2016; 11: 711-718.

26 Bourbeau J, Lavoie KL, Sedeno $\mathrm{M}$, et al. Behaviour-change intervention in a multicentre, randomised, placebo-controlled COPD study: methodological considerations and implementation. BMJ Open 2016; 6: e010109.

27 Troosters T, Bourbeau J, Maltais F, et al. Enhancing exercise tolerance and physical activity in COPD with combined pharmacological and non-pharmacological interventions: PHYSACTO randomised, placebo-controlled study design. BMJ Open 2016; 6: e010106.

28 Troosters T, Maltais F, Leidy N, et al. Effect of bronchodilation, exercise training, and behavior modification on symptoms and physical activity in chronic obstructive pulmonary disease. Am J Respir Crit Care Med 2018; 198: $1021-1032$.

29 Bourbeau J, Nault D, Sedeno M. Living well with COPD. www.livingwellwithcopd.com/en/publications.html. 2018 Date last accessed: July 12, 2018. 

change. J Consult Clin Psychol 1983; 51: 390-395.

31 Stern AF. The Hospital Anxiety and Depression Scale. Occup Med (Lond) 2014; 64: 393-394.

32 Olsson I, Mykletun A, Dahl AA. The Hospital Anxiety and Depression Rating Scale: a cross-sectional study of psychometrics and case finding abilities in general practice. BMC Psychiatry 2005; 5: 46.

33 Kroenke K, Spitzer RL, Williams JB. The PHQ-9: validity of a brief depression severity measure. J Gen Intern Med 2001; 16: 606-613.

34 Nasreddine ZS, Phillips NA, Bedirian V, et al. The Montreal Cognitive Assessment, MoCA: a brief screening tool for mild cognitive impairment. J Am Geriatr Soc 2005; 53: 695-699.

35 Kozora E, Tran ZV, Make B. Neurobehavioral improvement after brief rehabilitation in patients with chronic obstructive pulmonary disease. J Cardiopulm Rehabil 2002; 22: 426-430.

36 Etnier JL, Berry M. Fluid intelligence in an older COPD sample after short- or long-term exercise. Med Sci Sports Exerc 2001; 33: 1620-1628.

37 Coventry PA. Does pulmonary rehabilitation reduce anxiety and depression in chronic obstructive pulmonary disease? Curr Opin Pulm Med 2009; 15: 143-149.

38 Kanao K, Shiraishi M, Higashimoto Y, et al. Factors associated with the effect of pulmonary rehabilitation on physical activity in patients with chronic obstructive pulmonary disease. Geriatr Gerontol Int 2017; 17: 17-23.

39 Hamer M, Lavoie KL, Bacon SL. Taking up physical activity in later life and healthy ageing: the English Longitudinal Study of Ageing. Br J Sports Med 2014; 48: 239-243.

40 Northey JM, Cherbuin N, Pumpa KL, et al. Exercise interventions for cognitive function in adults older than 50: a systematic review with meta-analysis. Br J Sports Med 2018; 52: 154-160.

41 Chapman SB, Aslan S, Spence JS, et al. Distinct brain and behavioral benefits from cognitive vs. physical training: a randomized trial in aging adults. Front Hum Neurosci 2016; 10: 338.

42 Dustman RE, Ruhling RO, Russell EM, et al. Aerobic exercise training and improved neuropsychological function of older individuals. Neurobiol Aging 1984; 5: 35-42.

43 American Psychiatric Association. Diagnostic and Statistical Manual of Mental Disorders. Washington, DC, American Psychiatric Pub Inc., 2000.

44 Holas P, Michalowski J, Gaweda L, et al. Agoraphobic avoidance predicts emotional distress and increased physical concerns in chronic obstructive pulmonary disease. Respir Med 2017; 128: 7-12.

45 Swedish Council on Health Technology Assessment. Treatment of depression: a systematic review. www.ncbi.nlm. nih.gov/books/NBK447957/. 2004. Date last accessed: July 17, 2018.

46 Krishnan V, Nestler EJ. The molecular neurobiology of depression. Nature 2008; 455: 894-902.

47 Brewin CR. Theoretical foundations of cognitive-behavior therapy for anxiety and depression. Annu Rev Psychol 1996; 47: 33-57.

48 Knittle K, Nurmi J, Crutzen R, et al. How can interventions increase motivation for physical activity? A systematic review and meta-analysis. Health Psychol Rev 2018; 12: 211-230.

49 Petersen RC, Doody R, Kurz A, et al. Current concepts in mild cognitive impairment. Arch Neurol 2001; 58: 1985-1992.

50 van Beljouw IM, Verhaak PF, Cuijpers P, et al. The course of untreated anxiety and depression, and determinants of poor one-year outcome: a one-year cohort study. BMC Psychiatry 2010; 10: 86. 